\title{
ISSUES IN PUBLIC HEALTH
}

\section{Neonatal listeriosis during a countrywide epidemic in South Africa: A tertiary hospital's experience}

\author{
A Dramowski, ${ }^{1,2}$ MB ChB, PhD, FC Paed (SA), MMed (Paed), Cert Paed ID, DCH; L G Lloyd, ${ }^{1}$ MB ChB, FC Paed (SA), MMed (Paed), \\ Cert Neonatology (SA), DCH; A Bekker, ${ }^{1}$ MB ChB, PhD, FC Paed (SA), MMed (Paed), Cert Neonatology (SA); S Holgate, ${ }^{1}$ MB ChB, \\ FCPaed (SA), Cert Neonatology (SA), DCH (UK); M Aucamp, ${ }^{2}$ BSocSc (Nursing), PG Dipl IPC, MPhil HPE; K Reddy, ${ }^{3}$ MB ChB, \\ FC Path (SA) Micro, MMed (Microb Path), Dip Obst (SA), Dip HIV Man (SA), DMH (SA); H Finlayson, ${ }^{1}$ MB ChB, FC Paed (SA), \\ Cert ID (SA) Paed, DCH

\begin{abstract}
${ }^{1}$ Department of Paediatrics and Child Health, Faculty of Medicine and Health Sciences, Stellenbosch University, Cape Town, South Africa ${ }^{2}$ Academic Unit for Infection Prevention and Control, Division of Public Health and Health Systems, Department of Global Health, Faculty of Medicine and Health Sciences, Stellenbosch University, Cape Town, South Africa

${ }^{3}$ Department of Medical Microbiology, Faculty of Medicine and Health Sciences, Stellenbosch University, Cape Town, South Africa; and National Health Laboratory Service, Tygerberg Hospital, Cape Town, South Africa
\end{abstract}

Corresponding author: A Dramowski (dramowski@sun.ac.za)

\begin{abstract}
Background. A countrywide epidemic of Listeria monocytogenes (LM) in South Africa began in the first quarter of 2017, rapidly becoming the world's largest LM outbreak to date.

Methods. We describe the clinical course of neonates with culture-confirmed LM infection admitted to a tertiary neonatal unit at Tygerberg Hospital, Cape Town (1 January 2017 - 31 January 2018). Current epidemic LM cases were compared with a historical cohort of sporadic neonatal LM cases at our institution (2006 - 2016). The global literature on epidemic neonatal LM outbreaks (1 January 1978 - 31 December 2017) was reviewed.

Results. Twelve neonates (median gestational age 35 weeks, median birth weight $2020 \mathrm{~g}$ ) were treated for confirmed LM bacteraemia in 2017/18, presenting at a median age of 0.5 days. In 5 cases, neurolisteriosis was suspected. Three neonates died (25.0\%) v. 8/13 neonatal deaths $(61.6 \%)$ in the sporadic listeriosis cohort $(2006$ - 2016) $(p=0.075)$. The institution's neonatal LM infection incidence increased significantly in 2017 from a historical rate of $0.17 / 1000$ live births to 1.4/1 $000(p<0.001)$. During the current LM epidemic, the crude neonatal fatality rate exceeded the average calculated global epidemic neonatal LM mortality (3/12 (25.0\%) v. 50/290 (17.2\%); $p=0.448)$. Possible factors contributing to the high mortality rate in this epidemic LM neonatal cohort may include more virulent disease associated with sequence type 6 and the predominance of early-onset disease.

Conclusions. Epidemic neonatal listeriosis at Tygerberg Hospital was associated with a predominance of bacteraemic, early-onset disease. Listeriosis-associated mortality rates were higher than previously published, but lower than the rate in a historical institutional cohort.
\end{abstract}

S Afr Med J 2018;108(10):818-827. DOI:10.7196/SAMJ.2018.v108i10.13207

Listeria monocytogenes (LM) is a food-borne pathogen that causes a range of clinical syndromes including self-limiting gastroenteritis, bacteraemia and central nervous system disease. In immunocompetent hosts, the disease profile is usually mild and self-limiting, whereas immunocompromised hosts (including pregnant women and newborns) suffer severe and invasive forms of listeriosis. Numerous case reports of sporadic and epidemic human LM infection have been published, mainly from high-income countries. ${ }^{[1-4]}$ Of the seven known LM serotypes, four infect humans; historically, serotype $4 \mathrm{~b}$ has been the most common serotype associated with LM outbreaks. ${ }^{[5,6]}$

In South Africa (SA), epidemic listeriosis was first reported from Johannesburg in 1977/78, with 14 individuals infected (9 neonates and 5 adults) and an overall mortality rate of $43 \% \cdot{ }^{[7]}$ In the following four decades, few sporadic LM cases and suspected clusters were reported. ${ }^{[8,9]}$ Owing to the low background incidence of infection, invasive LM infection was not previously a notifiable disease in SA. In the first quarter of 2017, a countrywide increase in laboratoryconfirmed LM infections was noted, prompting a large-scale public health investigation by the National Institute of Communicable Diseases (NICD) to determine the source(s) of infection, the LM sequence-type(s) involved and the profile of affected cases. As at 9 April 2018, 1011 laboratory-confirmed cases of LM had been documented, with a mortality rate of $28 \%$ among cases with a known outcome (193/691). Most cases to date have been reported from Gauteng (59\%), Western Cape (12\%) and KwaZulu-Natal (7\%) provinces. Of cases with documented age, 41\% (418/1 011) were neonates aged $\leq 28$ days, most of whom (96\%) experienced disease onset in the first week of life. ${ }^{[10]}$ On 4 March 2018, the NICD announced that the source of the outbreak had been identified as ready-to-eat processed meat products manufactured at the Enterprise Foods Polokwane production facility, and a countrywide recall of the implicated food products began. Although the LM incidence rate has declined dramatically, a further 43 outbreakrelated cases have been confirmed since the recall owing to the long disease incubation period, a long refrigeration shelf-life of the contaminated products and the possibility of cross-contamination of other types of foods in the retail or home setting. ${ }^{[10]}$

Neonates with LM infection present with severe disease and experience high mortality rates. ${ }^{[11,12]}$ Most cases of neonatal listeriosis present within 7 days of birth (early-onset disease), although cases can occur up to 90 days of life (so-called late-onset disease). Early- 
onset infection tends to present with bacteraemia and higher case fatality rates, whereas late-onset disease is more likely to cause meningitis and central nervous system sequelae. ${ }^{[11]}$ In Cape Town's Metro East area, newborns with severe bacterial infection or other conditions requiring tertiary-level care are referred to the Tygerberg Hospital neonatal service. Of the 34 reported neonatal listeriosis cases in the Western Cape to date, $12(35 \%)$ were managed at Tygerberg Hospital. ${ }^{[10]}$

\section{Objectives}

In this report, we describe the clinical course and outcomes of the 12 neonates with epidemic listeriosis treated at Tygerberg Hospital, comparing them with a historical institutional cohort of sporadic neonatal LM cases and international publications reporting epidemic neonatal LM.

\section{Methods \\ Study setting}

Tygerberg Hospital in Cape Town, SA, is a tertiary academic medical complex with 1384 beds, including 300 neonatal and paediatric beds. The neonatal unit (124 beds) incorporates six clinical areas: an 8-bed combined medical/surgical neonatal intensive care unit (NICU), a 4-bed neonatal high-care unit, 2 acute neonatal wards, 1 low-care neonatal ward and 1 kangaroo mother care ward. There are $\sim 8000$ births ${ }^{[13]}$ and $\sim 2000$ neonatal ward admissions to Tygerberg Hospital annually, including both inborn babies and ill neonates $(<10$ days of age) transferred in from peripheral hospitals. The antenatal HIV prevalence rate is $\sim 17 \%$, and the low birth weight rate $(<2500 \mathrm{~g})$ was $37 \%$ in $2017 .^{[13]}$

\section{Investigation for suspected neonatal sepsis}

Well preterm ( $<37$ completed weeks) and term neonates with maternal indications for sepsis work-up (e.g. chorioamnionitis, spontaneous preterm labour) and ill neonates with any clinical, radiological and/or laboratory features suggesting infection underwent at least one blood culture with/without accompanying cerebrospinal fluid (CSF) specimens at the discretion of attending clinicians. Symptoms and signs that triggered investigation for sepsis included lethargy, apnoea, need for increased respiratory support, poor feeding, temperature instability, abdominal distension and a raised white cell count or C-reactive protein (CRP) level, among others. The unit's empirical antibiotic therapy regimen for early-onset neonatal sepsis is ampicillin plus gentamicin, and for early-onset meningitis cefotaxime plus ampicillin.

\section{Blood culture sampling and laboratory analysis}

Blood cultures and/or CSF samples were collected using an aseptic technique and submitted to the National Health Laboratory Service (NHLS) microbiology laboratory at Tygerberg Hospital. Blood cultures were incubated at $37^{\circ} \mathrm{C}$ using the BacT/Alert 3D Microbial Identification System (bioMérieux, France). On flagging positive, an aliquot of the blood culture broth was used to perform a Gram stain. In all cases (including the 12 neonatal LM cases reported here, where small Gram-positive bacilli were observed on the Gram stain) the microbiology laboratory promptly phoned out results to the clinicians. An aliquot of the broth was plated onto blood agar plates and incubated in a $\mathrm{CO}_{2}$ incubator at $35^{\circ} \mathrm{C}$ overnight, and a bile aesculin agar was inoculated. Colonies that appeared betahaemolytic on blood agar and hydrolysed aesculin were further identified using a catalase test and the Vitek 2 automated system (bioMérieux). Discrepancies in identification were resolved using the
BD BBL Crystal Gram positive identification kit (BD, USA) or Vitek MS (bioMérieux). Penicillin Etests (bioMérieux) were performed to determine the minimum inhibitory concentrations (MICs) for the isolates. For CSF samples, a cell count, protein and glucose estimation were performed, followed by performance of a Gram stain and inoculation of blood and cooked blood agar plates with overnight incubation in a $\mathrm{CO}_{2}$ incubator at $35^{\circ} \mathrm{C}$. A similar process to that described above for blood cultures was followed for identification of LM on CSF, resolution of discrepant results and sensitivity testing. Blood and CSF isolates confirmed as LM were submitted to the national reference laboratory at the NICD for sequence typing from mid-August 2017 onwards.

\section{Neonatal sepsis and LM outbreak surveillance and management}

The Unit for Infection Prevention and Control conducted routine surveillance for bloodstream infections (including early-onset neonatal sepsis and LM bacteraemia) on the Tygerberg Hospital neonatal platform in 2017/18. After declaration of the LM outbreak by the National Department of Health, mandatory reporting of LM infections to the provincial communicable disease control was introduced. For each case, additional demographic and outcome data, a case investigation form (including a history of the mother's food intake during pregnancy) and a clinical specimen for sequence typing was submitted to the NICD. The infection prevention nurse practitioner at our institution co-ordinated reporting of all LM cases and communication with hospital staff regarding affected patients. In view of several published cases of nosocomial transmission of LM infection to neonates, ${ }^{[14-17]}$ an alert to attending clinicians was distributed in January 2018 recommending use of contact precautions for LM-infected patients.

\section{Literature search terms}

We searched PubMed, African Journals Online and Google Scholar using the terms 'neonate, 'pregnancy', 'listeria,' 'listeriosis', 'neurolisteriosis' and 'outbreaks' for articles published from 1 January 1978 to 31 January 2017. We excluded publications that described sporadic neonatal LM infections only and epidemics with fewer than five neonatal cases reported. Each publication or outbreak database record was reviewed to extract the following information (when available): year/s reported, country, number of neonates infected, mortality rate and predominant serotype/s or sequence type identified.

\section{Study design}

Neonatal bloodstream infection episodes occurring between 1 January 2017 and 31 January 2018 were prospectively identified and recorded during routine surveillance activities. This dataset was searched to identify neonatal LM infections and cross-checked against neonatal admission records and the NHLS laboratory list of LM isolates on blood and CSF cultures. For calculation of LM infection incidence rates (historical and current), we divided the number of neonatal LM infections managed at the Tygerberg neonatal unit per year by the number of live births at the hospital in the same year. Additional demographic data on disease presentation, clinical course and therapy were obtained by neonatal folder review. Clinical data on the historical cohort of sporadic neonatal LM cases were obtained from a recently completed study in our department. ${ }^{[18]}$

Data handling, statistical analysis and ethical approval

For comparison of patient demographics and outcomes between the historical cohort and the current epidemic LM cases, we used Student's 
$t$-tests and Fisher's exact tests or $\chi^{2}$ tests for analysis of continuous and categorical variables, respectively. A $p$-value of $<0.05$ was considered statistically significant. Stata statistical software version 13.1 (StataCorp, USA) was used. Ethical approval and waiver of individual informed consent were obtained from the Human Health Research Ethics Committee of Stellenbosch University (ref. no. S13/09/171).

\section{Results \\ Epidemiology of neonatal LM infections at Tygerberg Hospital}

Between 2006 and 2016, sporadic LM infection was confirmed in 13 infants treated on the neonatal wards, ${ }^{[18]}$ with a mean institutional annual neonatal listeriosis rate of $0.17 / 1000$ live births. From 1 January 2017 to 31 January 2018, 12 cultureconfimed neonatal listeriosis cases were managed on the neonatal platform during the countrywide Listeria epidemic, with a neonatal listeriosis rate of $1.4 / 1000$ live births in 2017, representing a significant increase from the preceding period $(p<0.001)$ (Fig. 1). In comparison with other neonatal bloodstream infection pathogens by quarter of 2017, the proportion of infections caused by LM increased significantly in quarters 3 and 4, equalling or exceeding other earlyonset neonatal sepsis pathogens (Fig. 2).

\section{Case series of neonatal LM during the 2017 nationwide epidemic}

Twelve mothers from geographically diverse areas in the Cape Metro gave birth to 13 newborns (1 set of twins) (Table 1). LM bacteraemia was confirmed by culture in $12 / 13$ infants, although all received therapy for listeriosis. The mothers' median age was 31 years (interquartile range (IQR) 29 - 35). Caesarean sections were performed in 5/12 cases (41.7\%). Eight of 12 mothers (66.7\%) delivered at Tygerberg Hospital. Only 2/12 mothers (16.7\%) were HIV-positive (with viral loads of 1345 copies/mL and lower than detectable levels, respectively). Among the $9 / 12$ mothers $(75.0 \%)$ who went into spontaneous preterm labour, receipt of intrapartum antibiotics was documented in 6/9 $(66.7 \%)$ and unknown in the remaining 3. One mother was diagnosed with chorioamnionitis, 2 mothers had urinary tract infections and 1 mother had reported diarrhoea and decreased fetal movements 1 day prior to delivery. Histology reports available on 2 placentas showed macroscopic calcifications. No cases of fetal loss or stillbirth following maternal listeriosis were identified, although there is currently no

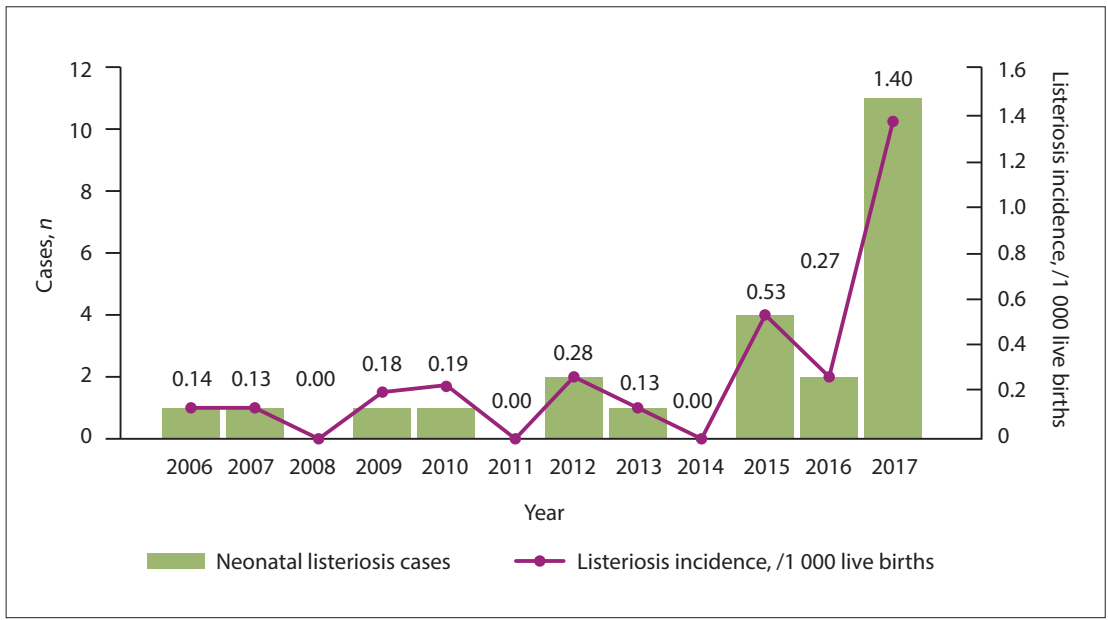

Fig. 1. Neonatal listeriosis rate at Tygerberg Hospital (sporadic cases $2006-2016$ and epidemic cases 2017). The annual LM incidence was calculated as the number of neonatal LM cases managed at Tygerberg Hospital per year/the total live births at the hospital in that year $\times 1000$ (historic cohort: 13/75 $195 \times 1000=0.17 / 1000$ live births; epidemic cohort up to 31 December 2017: 11/7 849 $\times$ $1000=1.40 / 1000$ live births $).(L M=$ Listeria monocytogenes. $)$

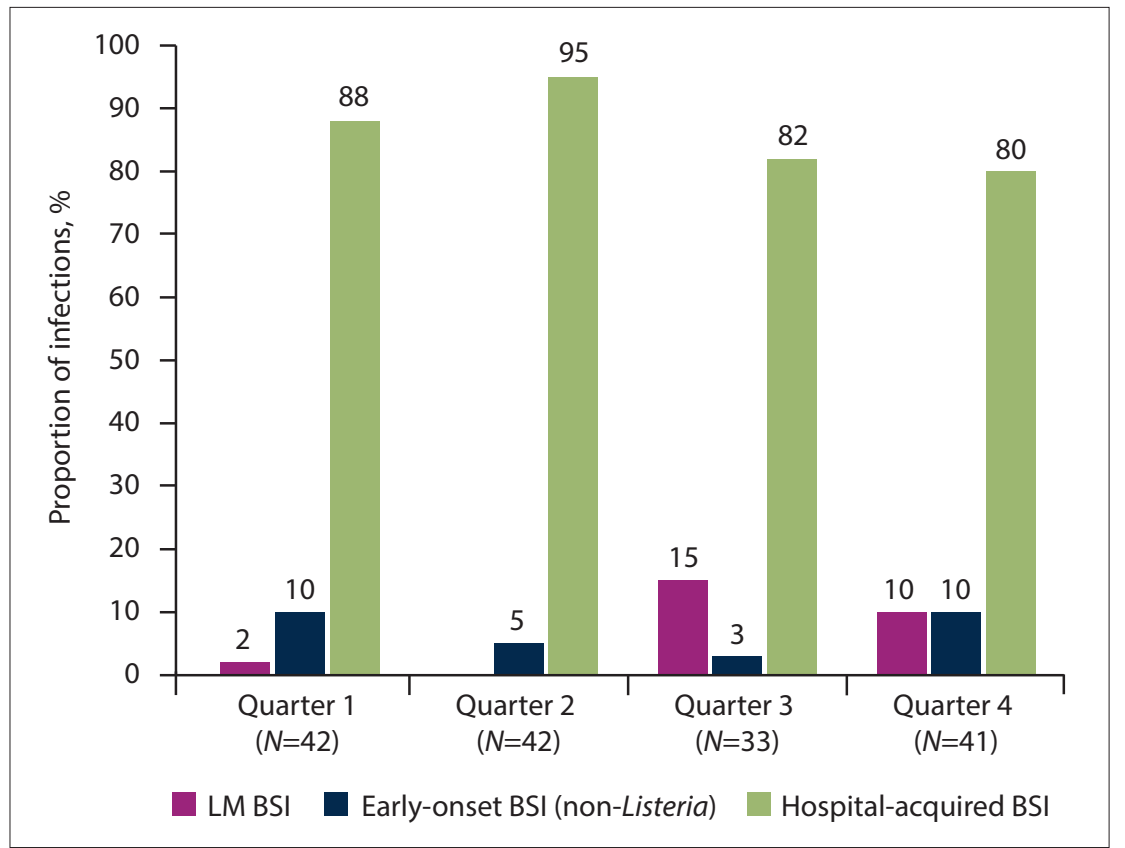

Fig. 2. Spectrum of neonatal BSIs at Tygerberg Hospital by quarter (2017). Non-listeria BSI included group B streptococci and Escherichia coli infections; hospital-acquired BSI were BSI pathogens identified on a blood culture specimen obtained $\geq 48$ hours after admission. ( $L M=$ Listeria monocytogenes; $B S I=$ bloodstream infection.

standardised protocol for investigation for LM infection in such cases at our institution. None of the mothers whose infants had culture-confirmed LM infection had growth of LM from clinical specimens.

\section{Neonates}

The median gestational age for the 12 newborns with culture-confirmed listeriosis (Table 1) was 35 weeks (IQR 33 - 38) and the median birth weight was $2020 \mathrm{~g}$ (IQR 1635 - 2 810) (Table 2). A 13th baby (twin of a baby with culture-confirmed LM) had respiratory distress at birth and an elevated CRP level $(61 \mathrm{mg} / \mathrm{dL})$ and was fully treated for LM infection, although blood and CSF cultures were negative.

Of the 12 babies with culture-confirmed LM, 9 (75.0\%) were premature and of low birth weight $(<2500 \mathrm{~g})$. All newborns had growth of LM from blood cultures within the first 5 days of life, with 6 positive cultures $(50.0 \%)$ on the first day of life. Lumbar punctures were performed in all but 


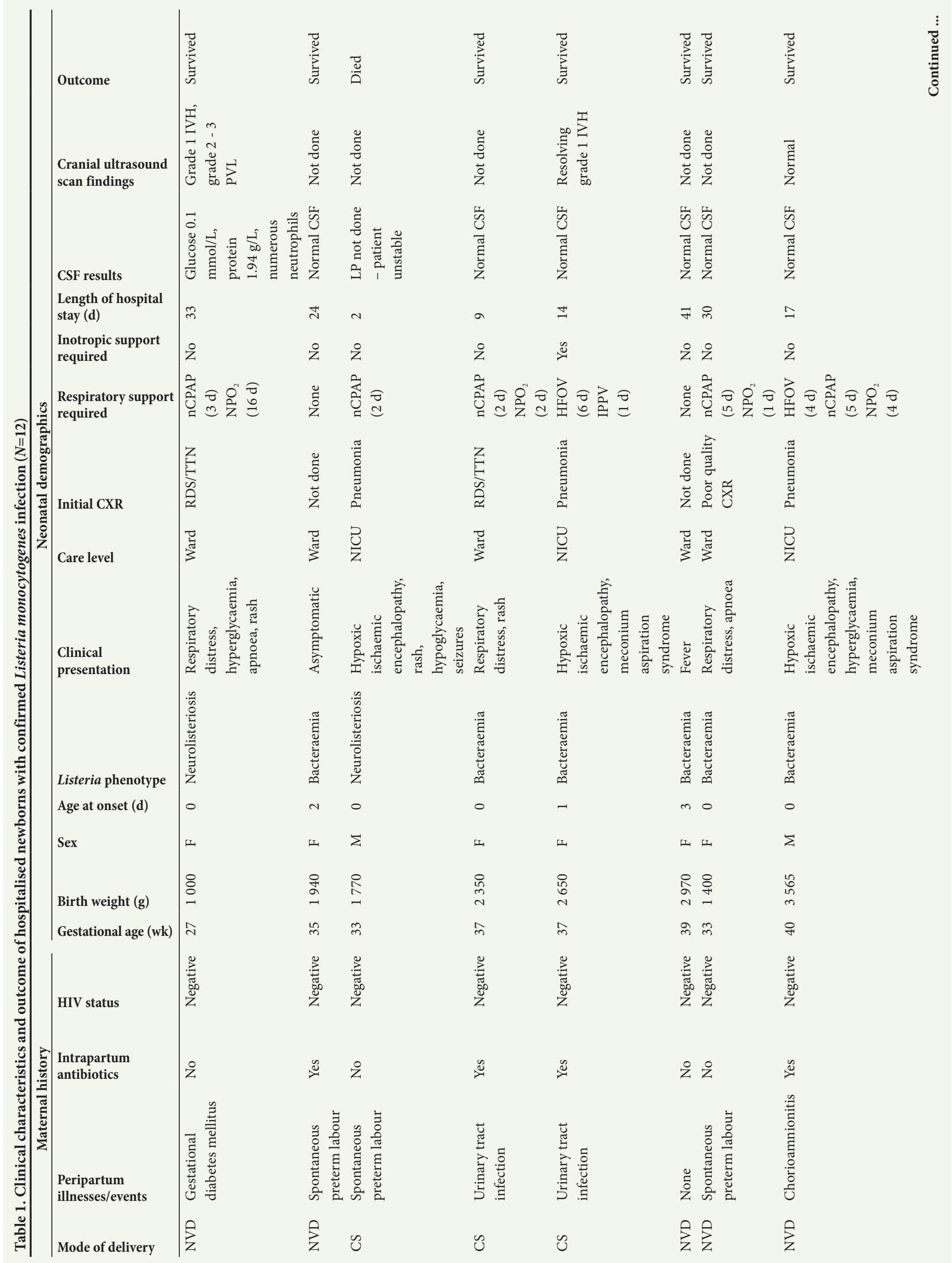




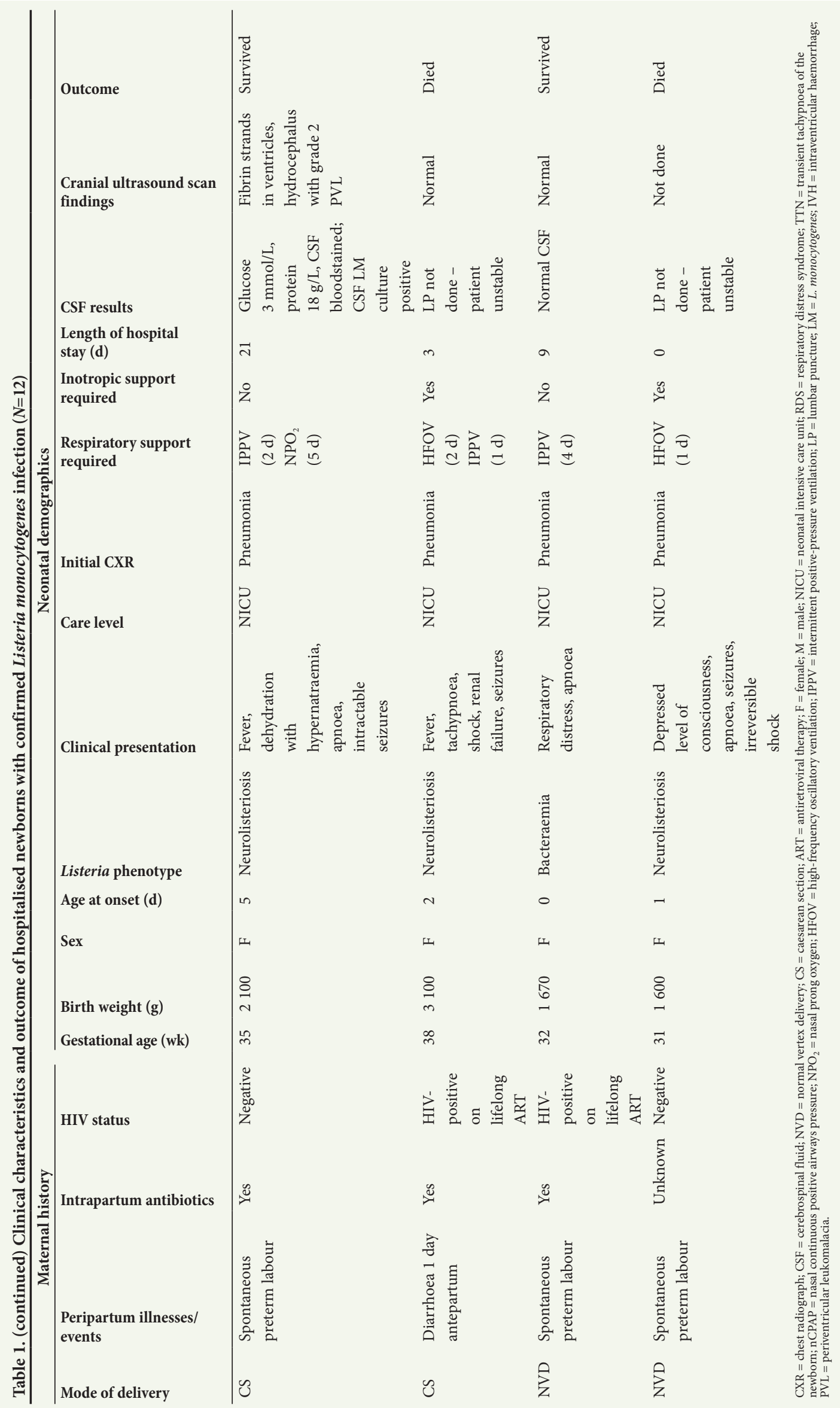


Table 2. Comparison of historical v. epidemic neonatal Listeria cohorts

\begin{tabular}{|c|c|c|c|c|}
\hline & $\begin{array}{l}\text { Total neonatal cases } \\
(2006-2018)(N=25)\end{array}$ & $\begin{array}{l}\text { Historical cohort } \\
(2006-2016)(N=13)\end{array}$ & $\begin{array}{l}\text { Epidemic cohort (Jan } \\
2017 \text { - Jan 2018) }(N=12)\end{array}$ & $p$-value \\
\hline Gestational age (wk), median (IQR) & $35(32-36)$ & $33(32-35)$ & $35(32.5-37.5)$ & 0.324 \\
\hline Birth weight (g), median (IQR) & $2100(1600-2500)$ & $2200(1500-2400)$ & $2020(1635-2810)$ & 0.531 \\
\hline Age at presentation (d), median (IQR) & $1(0-2)$ & $1(0-3)$ & $0.5(0-2)$ & 0.563 \\
\hline Length of stay* $(d)$, median (IQR) & $19(12-30)$ & $17(12-20)$ & $21(14-30)$ & 0.516 \\
\hline Age at death (d), median (IQR) & $2(1-5)$ & $2(1-4.5)$ & $2(1-5)$ & 0.916 \\
\hline Small for gestational age, $n(\%)$ & $6(24.0)$ & $4(30.8)$ & $2(16.7)$ & 0.363 \\
\hline Gender male, $n(\%)$ & $8(32.0)$ & $6(46.2)$ & $2(16.7)$ & 0.125 \\
\hline Place of birth peripheral hospital, $n(\%)$ & $14(56.0)$ & $10(76.9)$ & $4(33.3)$ & 0.036 \\
\hline HIV-exposed, $n(\%)$ & $6(24.0)$ & $4(30.8)$ & $2(16.7)$ & 0.363 \\
\hline Presenting symptom complex, $n(\%)$ & & & & 0.039 \\
\hline Respiratory & $9(36.0)$ & $4(30.8)$ & $5(41.6)$ & \\
\hline Neurological & $2(8.0)$ & 0 & $2(16.7)$ & \\
\hline Nonspecific & $2(8.0)$ & 0 & $2(16.7)$ & \\
\hline Combined & $12(48.0)$ & $9(69.2)$ & $3(25.0)$ & \\
\hline Highest level of neonatal care required, $n(\%)$ & & & & 0.073 \\
\hline Intensive care unit & $19(76.0)$ & $12(92.3)$ & $7(58.3)$ & \\
\hline High-care unit & $6(24.0)$ & $1(7.7)$ & $5(41.7)$ & \\
\hline Respiratory support required, $n$ (\%) & & & & 0.088 \\
\hline None & $2(8.0)$ & 0 & $2(16.7)$ & \\
\hline nCPAP & $5(20.0)$ & $1(7.7)$ & $4(33.3)$ & \\
\hline IPPV & $9(36.0)$ & $7(53.8)$ & $2(16.7)$ & \\
\hline HFOV & $9(36.0)$ & $5(38.5)$ & $4(33.3)$ & \\
\hline Inotropic support required, $n(\%)$ & $13(52.0)$ & $10(76.9)$ & $3(25.0)$ & 0.013 \\
\hline Neuroimaging findings, $n$ (\%) & & & & 0.387 \\
\hline Normal & $3(12.0)$ & 0 & $3(25.0)$ & \\
\hline IVH and/or PVL & $4(16.0)$ & $2(15.4)$ & $2(16.7)$ & \\
\hline Hydrocephalus & $4(16.0)$ & $3(23.1)$ & $1(8.3)$ & \\
\hline No neuroimaging done & $14(56.0)$ & $8(61.5)$ & $6(50.0)$ & \\
\hline Outcome death, $n$ (\%) & $11(44.0)$ & $8(61.5)$ & $3(25.0)$ & 0.075 \\
\hline
\end{tabular}

the 3 newborns who died ( 2 were too unstable and 1 had severe thrombocytopenia). Neurolisteriosis was clinically suspected in 5/12 newborns (41.7\%); however, only a single CSF sample cultured LM.

Half of the cohort required invasive ventilatory support and 3 needed inotropic support. Ten babies had a chest radiograph taken on admission: 7/10 had changes in keeping with pneumonia, 2/10 had features of transient tachypnoea of the newborn, and 1/10 was of poor quality (unable to interpret). Fifty percent (6/12) of the babies had cranial ultrasound scans. Half of these babies (3/6) had scans that were normal for their gestational age. Three babies had abnormalities detected. One had resolving grade 1 intraventricular haemorrhage (IVH), and another grade 1 IVH with grade 2 - 3 periventricular leukomalacia (PVL). The third baby had two scans, the initial one showing fibrin strands in the lateral ventricles (day 6) and a subsequent one demonstrating ventriculomegaly in keeping with hydrocephalus, as well as grade 2 PVL (day 15).

All 12 babies underwent laboratory investigations for sepsis on hospital admission, with the following results: median CRP $70 \mathrm{mg} /$ dL (IQR 14 - 298), median white cell count $10 \times 10^{9} / \mathrm{L}$ (IQR 8 - 15) and median platelet count $169 \times 10^{9} / \mathrm{L}$ (IQR $124-241$ ). All 12 newborns were commenced empirically on intravenous ampicillin and gentamicin on hospital admission; those who survived completed a total of 21 days on ampicillin, with an initial 7 days of gentamicin. In 10/12 cultures where MIC determination was performed, the MIC to penicillin was $<0.5 \mu \mathrm{g} / \mathrm{mL}$, i.e. susceptible. Only 2 of the 12 neonatal cases' specimens were processed at the NICD for sequence typing; both belonged to sequence type 6 (ST-6), which has been identified in $>90 \%$ of the SA LM epidemic isolates. ${ }^{[10]}$

\section{Comparison with the historical cohort}

From 2006 to 2016, 13 sporadic cases of listeriosis were managed on the Tygerberg Hospital neonatal wards ${ }^{[18]}$ v. 12 epidemic neonatal Listeria cases in 2017/18. There was only a single statistically significant difference between the cohorts (Table 2): the epidemic cohort had a higher incidence of neurolisteriosis and mixed respiratory/ neurological presentations. The sporadic cases experienced a higher case fatality rate $(62 \%$ v. $25 \%$; $p=0.075)$, had a higher proportion of neonates admitted to the NICU ( $92 \%$ v. $58 \% ; p=0.073)$ and included more infants who required ventilation $(92 \%$ v. $50 \% ; p=0.088)$, although these differences did not achieve statistical significance. In the historical cohort, 9/13 infants (69.2\%) received appropriate empirical cover for LM infection with ampicillin (v. 12/12 (100\%) of the epidemic cohort). When analysing the combined historical and epidemic cohort $(N=25)$, factors associated with neonatal LM-associated mortality on univariate analysis were being part of the historical cohort, need for NICU admission, need for inotropic 
support, and high-frequency oscillatory ventilation. In a multivariate regression analysis, the requirement for inotropic support was the only significant factor predicting mortality (Table 3 ).

Publications reporting neonatal listeriosis (1978 - 2017) We identified 15 publications that met our search criteria (Table 4). A single report from Africa was identified (in a large teaching hospital in SA). ${ }^{[7]}$ Most LM outbreaks described occurred in the 1980 s, with only two outbreaks involving neonates described since 1990. ${ }^{[28,29]}$ The largest outbreak affected 142 individuals, ${ }^{[2]}$ although most reports described small-scale epidemics and one described both epidemic and sporadic LM cases affecting neonates. ${ }^{[4]}$ Four publications reported neonatal cases only; in the articles describing mixed populations, neonates generally made up at least one-third of the cases. Overall mortality in neonatal listeriosis was high (mean
$17 \%$, range $0-44 \%)$. Of publications that reported the LM outbreak serotype involved, $1 \mathrm{a} / \mathrm{b}$ and $4 \mathrm{~b}$ were most prevalent.

\section{Discussion}

We present the only case series of epidemic neonatal listeriosis reported from Africa, and compare this cohort with historical sporadic neonatal LM cases at our institution and the international literature. The only other African case series of epidemic listeriosis (adults and neonates) reporting clinical data was also published from SA, nearly four decades ago, and involved 9 neonates. ${ }^{[7]}$ The cases reported here represent one-third of the neonatal cases identified in our province (Western Cape). ${ }^{[10]}$

The mean pre-epidemic, or historical, incidence of sporadic neonatal listeriosis at our institution (2006 - 2016) was 0.17 cases per 1000 live births (annual range 0 - 0.5), substantially exceeding

Table 3. Factors associated with mortality from neonatal listeriosis (combined historical and epidemic cohorts, $N=25)^{*}$

\begin{tabular}{|c|c|c|c|c|c|c|}
\hline \multirow[b]{2}{*}{ Factor } & \multirow{2}{*}{$\begin{array}{l}\text { Survived } \\
(N=14)\end{array}$} & \multirow[b]{2}{*}{ Died $(N=11)$} & \multirow{2}{*}{$\begin{array}{l}\text { Univariate } \\
\text { analysis, } \\
p \text {-value }\end{array}$} & \multicolumn{3}{|c|}{ Multivariate analysis } \\
\hline & & & & OR & 95\% CI & $p$-value \\
\hline Gestational age (wk), median (IQR) & $34(32-37)$ & $35(31-36)$ & 0.847 & - & - & - \\
\hline Birth weight in (kg), median (IQR) & $1.8(1.2-2.6)$ & $2.3(1.8-2.5)$ & 0.311 & - & - & - \\
\hline Age at presentation $(\mathrm{d})$, median (IQR) & $0.5(0-2)$ & $2.0(0-3)$ & 0.180 & - & - & - \\
\hline Cohort group (historical), $n(\%)$ & $5(35.7)$ & $8(72.7)$ & 0.075 & 1.1 & $0.09-13.9$ & 0.940 \\
\hline SGA, $n(\%)$ & $4(28.6)$ & $2(18.2)$ & 0.452 & - & - & - \\
\hline Gender (male), $n(\%)$ & $9(64.3)$ & $8(72.7)$ & 0.496 & - & - & - \\
\hline Place of birth (Tygerberg Hospital), $n$ (\%) & $6(42.9)$ & $8(72.7)$ & 0.138 & - & - & - \\
\hline $\begin{array}{l}\text { Ampicillin included in empirical antibiotic therapy } \\
\text { regimen, } n(\%)\end{array}$ & $13(92.9)$ & $9(81.8)$ & 0.564 & - & - & - \\
\hline HIV exposure status (HIV-exposed), $n$ (\%) & $4(28.6)$ & $2(18.2)$ & 0.452 & - & - & - \\
\hline Highest level of care (NICU), $n(\%)$ & $8(57.1)$ & $11(100)$ & 0.017 & $\dagger$ & $\dagger$ & + \\
\hline Respiratory support (oscillation), $n$ (\%) & $2(14.3)$ & $7(63.6)$ & 0.016 & + & $\dagger$ & + \\
\hline Inotropic support (required), $n(\%)$ & $3(21.4)$ & $10(90.9)$ & 0.001 & 34.9 & $2.3-532.1$ & 0.011 \\
\hline
\end{tabular}

Table 4. Published epidemic listeriosis events affecting neonates (1978 - 2017)

\begin{tabular}{|c|c|c|c|c|c|c|c|}
\hline Author, year of publication & Country & $\begin{array}{l}\text { Epidemic } \pm \text { sporadic } \\
\text { cases }\end{array}$ & $\begin{array}{l}\text { Year/s } \\
\text { reported }\end{array}$ & Cases, $N$ & $\begin{array}{l}\text { Liveborn } \\
\text { neonates, } \\
n(\%)\end{array}$ & $\begin{array}{l}\text { Neonatal } \\
\text { mortality } \\
\text { rate }^{\star}, n(\%)\end{array}$ & $\begin{array}{l}\text { Predominant } \\
\text { serotype } / \mathbf{s}^{\dagger}\end{array}$ \\
\hline Jacobs et al., ${ }^{[7]} 1978$ & SA & Epidemic & $1977-1978$ & 14 & $9(64.3)$ & $4 / 9(44.4)$ & $4 b$ \\
\hline Filice et al., ${ }^{[19]} 1978$ & USA & Epidemic & 1975 & 7 & $7(100.0)$ & 0 & $4 \mathrm{~b}$ \\
\hline Schlech et al., ${ }^{[20]} 1983$ & Canada & Epidemic & 1981 & 41 & $25(61.0)$ & $7 / 25(28.0)$ & $4 \mathrm{~b}$ \\
\hline Lennon et al., ${ }^{[21]} 1984$ & New Zealand & Epidemic & 1980 & 22 & $14(63.6)$ & $1 / 14(7.1)$ & $1 b$ \\
\hline Malinverni et al., ${ }^{[22]} 1985$ & Switzerland & Epidemic & $1983-1984$ & 25 & $11(44.0)$ & NR & $4 \mathrm{~b}$ \\
\hline Tulzer et al., ${ }^{[23]} 1987$ & Austria & Epidemic & 1986 & 20 & $20(100.0)$ & $5 / 20(25.0)$ & $1 / 2 \mathrm{a}$ \\
\hline Teberg et al., ${ }^{[24]} 1987$ & USA & Epidemic & 1986 & 23 & $23(100.0)$ & $5 / 23(21.7)$ & NR \\
\hline Linnan et al., 1988 & USA & Epidemic & 1985 & 142 & $93(65.5)$ & $10 / 93(10.8)$ & $4 \mathrm{~b}$ \\
\hline Mascola et al.,$^{[3]} 1989$ & USA & Epidemic & $1985-1986$ & 94 & $37(39.4)$ & $6 / 37(16.2)$ & $4 \mathrm{~b}$ and $1 \mathrm{a} / \mathrm{b}$ \\
\hline Bucher et al., ${ }^{[25]} 1989$ & Switzerland & Epidemic & $1983-1987$ & 35 & $35(100.0)$ & $5(14.3)$ & NR \\
\hline Allerberger et al., ${ }^{[26]} 1989$ & Austria & Epidemic & 1986 & 28 & $24(85.7)$ & $5 / 24(20.8)$ & NR \\
\hline Samuelsson et al., ${ }^{[27]} 1990$ & Denmark & Epidemic & $1985-1987$ & 35 & $8(22.9)$ & $1 / 8(12.5)$ & NR \\
\hline Frederiksen and Samuelsson, ${ }^{[4]} 1992$ & Denmark & Epidemic + sporadic & $1981-1988$ & 30 & $16(53.3)$ & $2 / 16(12.5)$ & 4 and 1 \\
\hline Elcuaz et al., ${ }^{[28]} 1996$ & Spain & Epidemic & $1991-1993$ & 24 & $7(29.2)$ & NR & 4 and 1 \\
\hline Pérez-Trallero et al., ${ }^{[29]} 2014$ & Spain & Epidemic & $2013-2014$ & 27 & $5(18.5)$ & 0 & $1 \mathrm{a} / \mathrm{b}$ and $4 \mathrm{~b}$ \\
\hline
\end{tabular}




\title{
INFECTION PREVENTION AND CONTROL ALERT, 26 JANUARY 2018
}

\author{
Listeriosis outbreak in South Africa \\ Implications for neonatal care at Tygerberg Hospital
}

Target group: Neonatal and obstetric healthcare professionals

By this time you would have heard of the nationwide outbreak of listeriosis in South Africa. Since 1 January 2017, 12 neonates in Tygerberg Hospital were treated for Listeria. See attached information sheet on listeriosis.

Although most of the babies acquire listeriosis from their mothers (transplacental transfer), research shows that it is possible for the organism, Listeria monocytogenes, to be transferred between neonates in healthcare. The transmission can already occur in the delivery room by means of respiratory equipment that is not cleaned properly after use, other contaminated instruments, multi-dose ointments or contaminated supplies.

\section{Practice Points}

- Contact precautions: Henceforth all neonates who test positive for Listeria must be placed on contact precautions until effective antibiotic treatment has been completed.

- Respiratory resuscitation equipment (laryngoscope handles, laryngoscope blades, introducers) must be cleaned meticulously between patients. Here are the steps for cleaning:

- Wash thoroughly with soap and water. Clean grooves and connection points with a brush.

- Rinse and dry.

- Wipe over with $70 \%$ alcohol.

Ideally the equipment must be sent to the CSSD for autoclaving.

- Hand hygiene: Contaminated hands always play an important role in the transfer of infection. Make therefore sure that you clean your hands between babies $100 \%$ of the time.

- Neonatal face masks (except the silicone ones) and their tubing are single-use items and must NOT be sent to the CSSD for reprocessing.

Please report all suspected listeriosis cases (expecting mothers, neonates) to the UIPC.

Fig. 3. Infection prevention clinical alert to obstetric and paediatric staff. (CSSD = Central Sterilisation Supply Department; UIPC = Unit for Infection Prevention and Control.)

rates reported from the UK, The Netherlands and the USA $(0.05$, 0.01 and 0.09 per 1000 live births, respectively).[30] Both the historical and epidemic listeriosis rates at our institution may be an underestimation, as these data reflect laboratory-confirmed cases only; some neonates may not have had lumbar punctures performed owing to clinical instability, others may have had antibiotics prior to blood/CSF culturing, and some may have died before the diagnosis was made.

A possible explanation for the apparently high historical rate of sporadic neonatal LM infection at Tygerberg Hospital is the use of the live births at this tertiary referral neonatal centre as the denominator, as opposed to the total population of the hospital's catchment area. An alternative hypothesis is that the high rate of 'sporadic' neonatal LM may include unrecognised prior outbreaks, as LM infections were not notifiable in SA before 2017. Other possible factors that may contribute to the comparatively high LM incidence in SA could include a more vulnerable population of pregnant women owing to high antenatal HIV prevalence, ${ }^{[13]}$ and a higher prevalence of LM-contaminated food and water sources than in high-income countries. ${ }^{[31-33]}$ Although HIV infection is not traditionally cited as a 
risk factor for listeriosis, it emerged as an important risk factor during the SA epidemic. ${ }^{[10]}$ However, in our epidemic cohort, only a single confirmed antenatal LM infection and two HIV-positive mothers of LM neonatal cases were identified.

Historically and during the current outbreak, LM bacteraemia events constitute a small percentage of the overall burden of bloodstream infections among hospitalised neonates at our institution. Even in the outbreak year (2017), the vast majority of neonatal bloodstream infections were hospital acquired, with LM cases peaking in quarter 3 at 15\% of all bacteraemia events. However, LM neonatal infections at our institution were associated with high mortality (sporadic $62 \%$ and outbreak $25 \%$ ), substantially exceeding the published crude fatality rate for neonatal nosocomial bloodstream infections at Tygerberg Hospital of $16 \%{ }^{[34]}$ Although $\mathrm{LM}$ is well documented as a nosocomial pathogen in neonates, ${ }^{[14-17]}$ we did not identify any nosocomial LM infections during 2017. Given the concerns regarding the potential for nosocomial LM transmission and the increasing incidence of neonatal infections in our unit, an infection control alert was issued to the hospital's obstetric and neonatal staff (Fig. 3).

Few major differences were observed between the historical and epidemic cohorts. In the epidemic cohort there was a larger proportion of babies with mixed respiratory and neurolisteriosis presentations, although only one baby cultured LM on CSF and just 3 of 6 cranial ultrasound scans performed had abnormal cranial ultrasound findings. However, 2 babies died before cranial ultrasound could be performed, and in the third baby who died, the scan was performed on day 4 of life, which may have been too early to detect all abnormalities. Although not reaching statistical significance owing to the small sample size, mortality was much lower in the epidemic cohort (25\% v. $62 \%)$, with a lower proportion of neonates requiring NICU admission, mechanical ventilation and inotropic support. In a multivariate analysis to identify factors associated with mortality from neonatal listeriosis (combined cohorts), the only factor that reached significance, with an odds ratio (OR) of 35, was inotropic support. However, this probably reflects the group of babies with refractory shock who had the most severe disease manifestations. In a recently published review of $>800$ cases of LM infection in neonates and adults (the MONALISA study), ${ }^{[1]}$ multiorgan failure was one of the strongest predictors of LM-associated mortality (OR 7.98, 95\% confidence interval 4.3 - 14.7).

In comparison with the global literature, our epidemic LM neonatal case fatality rate appears higher than average $(25 \%$ at Tygerberg Hospital v. 16\% globally). However, one-third of our cohort were severely ill neonates born at peripheral hospitals with delayed transfer to our NICU, which may have influenced disease outcome. In addition, Tygerberg Hospital is a tertiary referral facility caring for complicated obstetric and neonatal cases. Furthermore, all 12 babies presented with early-onset disease, which is known to be associated with poorer outcome. ${ }^{[11,12]}$ All neonates in the epidemic cohort received prompt, appropriate empirical antibiotic therapy including ampicillin and gentamicin, which is the locally recommended first-line therapy for early-onset neonatal sepsis. Of the laboratory investigations submitted at the time of sepsis work-up, CRP was elevated (>10 mg/dL) in $12 / 12$ babies (100\%), the platelet count was reduced $\left(<250 \times 10^{9} / \mathrm{L}\right)$ in $9 / 12(75.0 \%)$, and the total white cell count was normal $\left(8-25 \times 10^{9} / \mathrm{L}\right)$ in most $(10 / 12,83.3 \%)$. In this epidemic cohort, raised CRP and reduced platelet count appear to be the most useful markers of infection.

Only 2 patients in our cohort had sequence typing results available, confirming ST-6 disease, in keeping with $>90 \%$ of the SA LM outbreak isolates. Owing to technological advances in LM strain typing approaches, there is a paucity of data on ST-6's historical involvement in outbreaks. However, this sequence type is associated with a more severe disease course, ${ }^{[35]}$ and is an additional factor that may explain our higher neonatal fatality rate in comparison with the published literature. Despite intensive investigation by the NICD, no outbreak source/s for the current SA LM epidemic have been identified. Although food histories have been obtained from many affected patients, no obvious patterns in consumption have emerged, with potentially long intervals between consumption of an LM-contaminated foodstuff and disease onset. ${ }^{[10]}$

\section{Study strengths and limitations}

The strengths of this study include the use of a clear case definition (laboratory-confirmed listeriosis), the detailed description of the neonates' disease course, and comparison with an institutional cohort of sporadic neonatal LM cases. However, our study limitations include the small cohort size, possible underestimation of the epidemic listeriosis rate, limited data on maternal and stillbirthassociated LM infections, and description of a tertiary hospital cohort (representing the sickest neonates with LM who were born in or referred to our institution).

\section{Conclusions}

Epidemic neonatal listeriosis at Tygerberg Hospital was associated with a predominance of bacteraemic disease and severe respiratory complications. Listeriosis-associated mortality rates were higher than previously published, but lower than the rate in a historical institutional cohort. Although we did not identify any cases, the documented risk of nosocomial LM transmission warrants application of transmission-based precautions for LM cases during outbreaks.

Acknowledgements. The authors thank the NHLS, the NICD and the patients and staff of Tygerberg Hospital.

Author contributions. All authors contributed to the study design and data collection. $\mathrm{AB}, \mathrm{AD}$ and LGL completed the data analysis. $\mathrm{AD}$ produced the first draft of the manuscript. All authors critically reviewed the manuscript and approved the final version.

Funding. National Research Foundation rated researcher incentive fund. Conflicts of interest. None.

1. Charlier C, Perrodeau É, Leclercq A, et al; MONALISA study group. Clinical features and prognostic factors of listeriosis: The MONALISA national prospective cohort study. Lancet Infect Dis factors of listeriosis: The MONALISA national prospective conor
2017;17(5):510-519. https://doi.org/10.1016/S1473-3099(16)30521-7

2. Linnan MI, Mascola L, Lou XD, et al. Epidemic listeriosis associated with Mexican-style cheese. N Engl 2. Linnan MJ, Mascola L, Lou XD, et al. Epidemic listeriosis associated with Mexic
J Med 1988;319(13):823-828. https://doi.org/10.1056/NEJM198809293191303

3. Mascola L, Sorvillo F, Neal J, Iwakoshi K, Weaver R. Surveillance of listeriosis in Los Angeles County 1985 - 1986. A first year's report. Arch Intern Med 1989;149(7):1569-1572.

4. Frederiksen B, Samuelsson S. Feto-maternal listeriosis in Denmark 1981 - 1988. J Infect 1992;24(3):277287. https://doi.org/10.1016/S0163-4453(05)80033-7

5. Pinner RW, Schuchat A, Swaminathan B, et al. Role of foods in sporadic listeriosis. II. Microbiologic and epidemiologic investigation: The Listeria Study Group. JAMA 1992;267(15):2046-2050. https:// doi.org/10.1001/jama.1992.03480150052036

6. Orsi RH, den Bakker HC, Wiedmann M. Listeria monocytogenes lineages: Genomics, evolution, ecology, and phenotypic characteristics. Int J Med Microbiol 2011;301(2):79-96. https.//dol org/10.1016/jijmm. 2010.05.002

7. Jacobs MR, Stein H, Buqwane A, et al. Epidemic listeriosis: Report of 14 cases detected in 9 months. S Afr Med Stein $\mathrm{H}$, Buqwane A,

Smith AM, Naicker P, Bamford C, et al. Genome sequences for a cluster of human isolates of Listeria

Smith AM, Naicker P, Bamford C, et al. Genome sequences for a cluster of human isolates of Listeria
monocytogenes identified in South Africa in 2015. Genome Announc 2016;4(2):e00200-16. https://doi. monocytogenes identified in South

org/10.1128/genomeA.00200-16
National Institute for Communicable Diseases. Communicable Diseases Communique 2015;14(11):10-11. https://www.mm3admin.co.za/documents/docmanager/47F686F5-ACDF-44628366-64AFD5633D81/00099413.pdf (accessed 19 February 2018).

10. National Institute for Communicable Diseases. Situation update on listeriosis outbreak, South Africa 13 April 2018. http://www.nicd.ac.za/index.php/nicd-listeriosis-situation-report-13-april-2018 (accessed 18 April 2018).

1. Bortolussi R. Listeria monocytogenes infections in neonates. Semin Pediatr Infect Dis 1999;10(2):111118. https://doi.org/10.1016/S1045-1870(99)80042-9

12. Posfay-Barbe KM, Wald ER. Listeriosis. Semin Fetal Neonatal Med 2009;14(4):228-233. https://doi. org/10.1016/j.siny.2009.01.006

13. Mason D, Gebhardt GS. PPIP data Cape Town Metro 2014 - 2016. 2017. http://www.obstyger.co.za/ Publications.html (accessed 3 November 2017) 
14. Tortajada C, Porta R, Riba M, Santoma MJ, Palacín E, Español M. [Nosocomial outbreak due to Listeric monocytogenes in a neonatal unit]. Enferm Infecc Microbiol Clin 2012;30(3):143-146. https://doi. monocytogenes in a neonatal unit.
org/10.1016/j.eimc.2011.07.018

15. Lazarus $C$, Leclercq A, Lecuit $M$, et al. Probable nosocomial transmission of listeriosis in neonates. J Hosp Infect 2013;85(2):159-160. https://doi.org/10.1016/j.jhin.2013.06.019

16. Fullerton L, Norrish G, Wedderburn CJ, Paget S, Basu Roy R, Cane C. Nosocomial neonatal Listeri monocytogenes transmission by stethoscope. Pediatr Infect Dis J 2015;34(9):1042-1043. https://doi org/10.1097/INF.000000000000078

17. Nelson KE, Warren D, Tomasi AM, Raju TN, Vidyasagar D. Transmission of neonatal listeriosis in a delivery room. Am J Dis Child 1985;139(9):903-905. https://doi.org/10.1001/ archpedi.1985.02140110057029

18. Oppel K. A retrospective review of Listeria monocytogenes infection at Tygerberg Children's Hospital from 2006 - 2016: Is empiric ampicillin still indicated beyond the early neonatal period? MMed Paediatrics dissertation. Cape Town: Stellenbosch University, 2018. http://scholar.sun.ac.za/ handle/10019.1/3785 (accessed 10 February 2018).

19. Filice GA, Cantrell HF, Smith AB, Hayes PS, Feeley JC, Fraser DW. Listeria monocytogenes infection in neonates: Investigation of an epidemic. J Infect Dis 1978;138(1):17-23. https://doi.org/10.1093/ infdis/138.1.17

20. Schlech WF Lavigne PM, Bortolussi RA, et al. Epidemic listeriosis - evidence for transmission by food. N Engl J Med 1983;308(4):203-206. https://doi.org/10.1056/NEJM198301273080407

21. Lennon D, Lewis B, Mantell C, et al. Epidemic perinatal listeriosis. Pediatr Infect Dis 1984;3(1):303Lennon

22. Malinverni R, Bille J, Perret C, Regli F, Tanner F, Glauser MP. [Epidemic listeriosis. Report of 25 cases in 15 months at the Vaud University Hospital Center]. Schweiz Med Wochenschr 1985;115(1):2-10.

23. Tulzer G, Bauer R, Daubek-Puza WD. [Local epidemic of neonatal listeriosis in Upper Austria - report of 20 cases]. Klin Padiatr 1987;199(5):325-328. https://doi.org/10.1055/s-2008-1026813

24. Teberg AJ, Yonekura ML, Salminen C, Pavlova Z. Clinical manifestations of epidemic neonatal listeriosis. Pediatr Infect Dis J 1987;6(9):817-820. https://doi.org/10.1097/00006454-198709000-00003

25. Bucher HU, Nadal D, Mieth D. [Listeriosis in the newborn infant: improved prognosis due to early detection]. Monatsschr Kinderheilkd 1989;137(6):321-325.

26. Allerberger F, Guggenbichler JP. Listeriosis in Austria - report of an outbreak in 1986. Acta Microbiol Hung 1989;36(2-3):149-152.
27. Samuelsson S, Rothgardt NP, Carvajal A, Frederiksen W. Human listeriosis in Denmark 1981 1987 including an outbreak November 1985 - March 1987. J Infect 1990;20(3):251-259. https://doi. org/10.1016/0163-4453(90)91244-8

28. Elcuaz R, Bordes A, Aladro Y, et al. [Clinical characteristics and epidemiologic study of a listeriosis outbreak in Grand Canary]. Enferm Infecc Microbiol Clin 1996;14(7):416-421.
out

29. Pérez-Trallero E, Zigorraga C, Artieda J, Alkorta M, Marimón JM. Two outbreaks of Listeria monocytogenes infection, Northern Spain. Emerg Infect Dis 2014;20(12):2155-2157. https://doi. org/10.3201/eid2012.140993

30. Okike IO, Lamont RF, Heath PT. Do we really need to worry about Listeria in newborn infants? Pediatr Infect Dis J 2013;32(4):405-406. https://doi.org/10.1097/INF.0b013e3182867fa0

31. Nyenje ME, Odjadjare CE, Tanih NF, Green E, Ndip RN. Foodborne pathogens recovered from ready-to-eat foods from roadside cafeterias and retail outlets in Alice, Eastern Cape Province, South Africa: Public health implications. Int J Environ Res Public Health 2012;9(8):2608-2619. https://doi, org $/ 10.3390 /$ ijerph 9082608

32. Odjadjare EE, Obi LC, Okoh AI. Municipal wastewater effluents as a source of listerial pathogens in the aquatic milieu of the Eastern Cape Province of South Africa: A concern of public health importance. Int J Environ Res Public Health 2010;7(5):2376-2394. https://doi.org/10.3390/ijerph7052376

33. Jongman M, Korsten L. Microbial quality and suitability of roof-harvested rainwater in rural villages for crop irrigation and domestic use. J Water Health 2016;14(6):961-971. https://doi.org/10.2166/
for for crop irrig
wh. 2016.058

34. Dramowski A, Madide A, Bekker A. Neonatal nosocomial bloodstream infections at a referral hospital in a middle-income country: Burden, pathogens, antimicrobial resistance and mortality. Paediatr Int Child Health 2015;35(3):265-272. https://doi.org/10.1179/2046905515Y.0000000029

35. Koopmans MM, Brouwer MC, Bijlsma MW, et al. Listeria monocytogenes sequence type 6 and increased rate of unfavorable outcome in meningitis: Epidemiologic cohort study. Clin Infect Dis 2013;57(2):247-253. https://doi.org/10.1093/cid/cit250

Accepted 23 April 2018 